\title{
Covariant Poisson Structures on Complex Projective Spaces
}

\author{
Albert JeU-Liang SheU ${ }^{1}$
}

\begin{abstract}
In this paper, we describe a one-parameter family of nonstandard $S U(n+1)$-covariant (also known as $S U(n+1)$-homogeneous) Poisson structures $\tau_{c}$ on the projective space $\mathbb{C} P^{n}$ that represents the classical counterpart of the quantum family $C\left(\mathbb{C} P_{q, c}^{n}\right)$, and show that the standard Poisson $\mathbb{S}^{2 n-1}$ is indeed embedded in each of these nonstandard Poisson $\mathbb{C} P^{n}$. We explicitly describe the Lagrangian subalgebra associated with such a Poisson homogeneous space. We also give an elementary proof of the statement that (non-zero) $S U(n)$-invaraint contravariant alternating 2-tensors on $\mathbb{S}^{2 n-1}$ with $n \neq 3$ (or $\mathbb{C} P^{n}$ ) are unique, up to a constant factor.
\end{abstract}

\section{Introduction.}

One of the most intriguing aspects of the theory of quantum groups and quantum spaces [Dr1, RTF, So, Wo1, Wo2, Po, Ri, VaSo] is the close interplay between the geometric structure of the underlying Poisson Lie group (or Poisson space) [We2, LuWe1] and the algebraic structure on the corresponding quantum group (or quantum space). For example, Soibelman's classification [So] of all irreducible *-representations of the quantum algebra $C\left(G_{q}\right)$ for compact Poisson simple Lie groups $G$ gives a one-to-one correspondence between irreducible ${ }^{*}$-representations of $C\left(G_{q}\right)$ and the symplectic leaves [We2] on $G$. This leads to a groupoid $\mathrm{C}^{*}$-algebraic [Re] approach to study the structure of the algebra $C\left(G_{q}\right)$ [Sh2] which shows that the decomposition of $S U(n)$ (or $\mathbb{S}^{2 n+1}$ ) by symplectic leaves of various dimensions corresponds to a compatible decomposition of $C\left(S U(n)_{q}\right)$ (or $C\left(\mathbb{S}_{q}^{2 n+1}\right)$ ) by its (closed) ideals in the spirit of noncommutative geometry.

Given a Poisson Lie group $G$ with Poisson structure $\pi$, we call a Poisson structure $\tau$ on a homogeneous space $M$ of $G$ a $(G, \pi)$-covariant [LuWe2] (also known as $(G, \pi)$-homogeneous [Dr2]) Poisson structure on $M$ if the

\footnotetext{
${ }^{1}$ Partially supported by NSF Grant DMS-9623008.
} 
$G$-action map $G \times M \rightarrow M$ is a Poisson map with respect to the product Poisson structure $\pi \oplus \tau$ on $G \times M$ and the Poisson structure $\tau$ on $M$. It is well known that the standard multiplicative Poisson structure on $S U(n+1)$ induces a standard covariant Poisson structure on the homogeneous spaces $\mathbb{S}^{2 n+1}=S U(n+1) / S U(n)$ and $\mathbb{C} P^{n}=S U(n+1) / U(n)$ determined by the Poisson Lie subgroups $S U(n)$ and $U(n)$, respectively. On the other hand, Lu and Weinstein [LuWe2] described explicitly all $S U(2)$ covariant Poisson structures on $\mathbb{S}^{2}=\mathbb{C} P^{1}$ including a one-parameter family of nonstandard $S U$ (2)-covariant Poisson structures on $\mathbb{S}^{2}$, and showed that each nonstandard covariant Poisson sphere $\mathbb{S}_{c}^{2}$ contains a copy of the trivial Poisson 1-sphere $\mathbb{S}^{1}$ (consisting of a circle family of 0-dimensional symplectic leaves) and exactly two 2-dimensional symplectic leaves. This geometric structure is reflected faithfully in the algebraic structure of the algebra $C\left(\mathbb{S}_{q c}^{2}\right)$ of the nonstandard quantum spheres $\mathbb{S}_{q c}^{2}[\mathrm{Sh} 1]$.

Dijkhuizen and Noumi studied in great detail [DiNo] a one-parameter family of nonstandard quantum projective spaces $\mathbb{C} P_{q, c}^{n}$ with quantum algebras $C\left(\mathbb{C} P_{q, c}^{n}\right)$. In [Sh3], the structure of $C\left(\mathbb{C} P_{q, c}^{n}\right)$ is studied and analyzed as a groupoid $\mathrm{C}^{*}$-algebra, and an algebraic decomposition of $C\left(\mathbb{C} P_{q, c}^{n}\right)$ by a closed ideal indicates that the underlying nonstandard projective space Poisson $\mathbb{C} P^{n}$ should contain an embeded copy of the standard Poisson $\mathbb{S}^{2 n-1}$. Using the result of [KhRaRu], one can classify all $S U(n+1)$-covariant Poisson structures on $\mathbb{C} P^{n}$ into a one-parameter family. In this paper, we describe the part of this one-parameter family of Poisson structures on $\mathbb{C} P^{n}$ that represents the classical counterpart of the quantum family $C\left(\mathbb{C} P_{q, c}^{n}\right)$, and show that the standard Poisson $\mathbb{S}^{2 n-1}$ is indeed embedded in each of these nonstandard Poisson $\mathbb{C} P^{n}$. Furthermore we find explicitly the Lagrangian subalgebras [Dr2] associated with these nonstandard $S U(n+1)$-covariant Poisson structures on $\mathbb{C} P^{n}$. We also give an elementary proof of the fact that (non-zero) $S U(n)$-invaraint (contravariant alternating) 2-tensors on $\mathbb{S}^{2 n-1}$ with $n \neq 3$ (or on $\mathbb{C} P^{n}$ ) are unique, up to a constant factor. We remark that in [KhRaRu], Khoroshkin, Radul, and Rubtsov obtained interesting results about covariant Poisson structures on coadjoint orbits, including $\mathbb{C} P^{n}$. Our approach, motivated by Dijkhuizen and Noumi's work [DiNo], is different from theirs and the embedding of the standard Poisson $\mathbb{S}^{2 n-1}$ in the nonstandard Poisson $\mathbb{C} P^{n}$ is new.

We would like to thank the referee for very helpful suggestions and comments. 


\section{Poisson structure on Lie groups.}

In this section, we discuss some basic properties of affine Poisson structures in the form needed later. We recall that an affine Poisson structure on a Lie group $G$ is given by a Poisson 2-tensor $\pi \in \Gamma\left(\wedge^{2} T G\right)$, such that

$$
\pi(g h)=L_{g}(\pi(h))+R_{h}(\pi(g))-L_{g} R_{h}(\pi(e))
$$

for any $g, h \in G$ [We3], or equivalently,

$$
\pi_{l}(g):=\pi(g)-L_{g}(\pi(e))
$$

for $g \in G$ defines a multiplicative Poisson 2-tensor on $G$ [Lu, DaSo], where $L_{g}$ and $R_{g}$ are the left and the right actions by $g \in G$, respectively, and $e$ is the identity element of $G$. For an affine Poisson 2-tensor $\pi$ on a Lie group $G$, the left action of the Poisson-Lie group $\left(G, \pi_{l}\right)$ on the Poisson manifold $(G, \pi)$ by left translation is a Poisson action, i.e., the multiplication map $G \times G \rightarrow G$ is a Poisson map, where $G \times G$ and $G$ are endowed with the Poisson structures $\pi_{l} \times \pi$ and $\pi$, respectively. In another word, $\pi$ on $G$ (as a homogeneous space of $G$ ) is a (left) $\left(G, \pi_{l}\right)$-covariant Poisson structure.

A typical example of an affine Poisson structure on a Poisson-Lie group $G$ with multiplicative Poisson 2-tensor $\pi$ is provided by a right translation $\pi_{\sigma}$ of $\pi$ by an element $\sigma \in G$, i.e.,

$$
\pi_{\sigma}(g):=R_{\sigma}\left(\pi\left(g \sigma^{-1}\right)\right)
$$

for $g \in G$. Since the right translation by $\sigma$ on $G$ is a diffeomorphism on $G$, the 'push-forward' $\pi_{\sigma}$ of $\pi$ by $R_{\sigma}$ is clearly a Poisson 2 -tensor on $G$. Furthermore,

$$
\begin{aligned}
\left(\pi_{\sigma}\right)_{l}(g) & =R_{\sigma}\left(\pi\left(g \sigma^{-1}\right)\right)-L_{g}\left(R_{\sigma}\left(\pi\left(\sigma^{-1}\right)\right)\right) \\
& =R_{\sigma}\left(L_{g}\left(\pi\left(\sigma^{-1}\right)\right)+R_{\sigma^{-1}}(\pi(g))\right)-L_{g}\left(R_{\sigma}\left(\pi\left(\sigma^{-1}\right)\right)\right) \\
& =R_{\sigma} L_{g}\left(\pi\left(\sigma^{-1}\right)\right)+\pi(g)-R_{\sigma} L_{g}\left(\pi\left(\sigma^{-1}\right)\right)=\pi(g)
\end{aligned}
$$

which is a multiplicative Poisson 2-tensor on $G$. So $\pi_{\sigma}$ on $G$ (as a homogeneous space of $G$ ) is a (left) $(G, \pi)$-covariant Poisson structure, for any $\sigma \in G$. Note that

$$
\pi_{\sigma}=\pi+\left(X_{\sigma}\right)^{l}
$$

where $X_{\sigma}:=\pi_{\sigma}(e)=R_{\sigma}\left(\pi\left(\sigma^{-1}\right)\right) \in \mathfrak{g} \wedge \mathfrak{g}$ and $X^{l}(g):=L_{g}(X)$ is the left-invariant 2-tensor generated by $X \in \mathfrak{g} \wedge \mathfrak{g}$, since $\left(\pi_{\sigma}\right)_{l}=\pi$. 
When a closed subgroup $H$ of a Lie group $G$ is coisotropic [We1] with respect to a Poisson structure $\rho$ on $G$, i.e.,

$$
\rho(g h)-R_{h}(\rho(g)) \in L_{g h}(\mathfrak{h} \wedge \mathfrak{g})
$$

for all $g \in G$ and $h \in H$, it is easy to see that the Poisson bracket $\left\{f_{1}, f_{2}\right\}:=$ $\left(d f_{1} \wedge d f_{2}\right)(\rho)$ of $f_{1}, f_{2} \in C^{\infty}(G / H) \subset C^{\infty}(G)$ is still in $C^{\infty}(G / H)$ and hence induces a Poisson structure on $G / H$, or equivalently, a Poisson 2tensor $\tilde{\rho}$ on the homogeneous space $G / H$ is well defined by

$$
\tilde{\rho}([g H]):=[\rho(g)] \in L_{g}\left(\wedge^{2}(\mathfrak{g} / \mathfrak{h})\right)=\wedge^{2} T_{[g H]}(G / H) .
$$

Given a Poisson-Lie group $(G, \pi)$ and $\sigma \in G$, if a closed subgroup $H$ of $G$ is coisotropic with respect to $\pi$, then $H$ is coisotropic with respect to the affine Poisson structure $\pi_{\sigma}$ on $G$ if and only if

$$
\left(X_{\sigma}\right)^{l}(g h)-R_{h}\left(\left(X_{\sigma}\right)^{l}(g)\right) \in L_{g h}(\mathfrak{h} \wedge \mathfrak{g})
$$

since $\pi_{\sigma}=\pi+\left(X_{\sigma}\right)^{l}$ and $\pi(g h)-R_{h}(\pi(g)) \in L_{g h}(\mathfrak{h} \wedge \mathfrak{g})$. Now

$$
\begin{aligned}
\left(X_{\sigma}\right)^{l}(g h)-R_{h}\left(\left(X_{\sigma}\right)^{l}(g)\right) & =L_{g h}\left(X_{\sigma}\right)-R_{h}\left(L_{g}\left(X_{\sigma}\right)\right) \\
& =L_{g}\left(L_{h} X_{\sigma}-R_{h} X_{\sigma}\right) \\
& =L_{g} L_{h}\left(X_{\sigma}-L_{h^{-1}} R_{h} X_{\sigma}\right) \\
& =L_{g h}\left(\mathrm{id}-\operatorname{Ad}_{h^{-1}}\right)\left(X_{\sigma}\right) .
\end{aligned}
$$

So $\left(X_{\sigma}\right)^{l}(g h)-R_{h}\left(\left(X_{\sigma}\right)^{l}(g)\right) \in L_{g h}(\mathfrak{h} \wedge \mathfrak{g})$ for all $(g, h) \in G \times H$ if and only if

$$
\left(\mathrm{id}-\operatorname{Ad}_{h^{-1}}\right)\left(X_{\sigma}\right) \in \mathfrak{h} \wedge \mathfrak{g}
$$

for all $h \in H$, or equivalently,

$$
\operatorname{ad}_{\mathfrak{h}}\left(X_{\sigma}\right) \subset \mathfrak{h} \wedge \mathfrak{g} .
$$

Thus we get the following result.

Proposition 1. Given a Poisson-Lie group $(G, \pi)$ and a closed subgroup $H$ of $G$ that is coisotropic with respect to $\pi$, the subgroup $H$ is coisotropic with respect to $\pi_{\sigma}$ for $\sigma \in G$, if and only if $\operatorname{ad}_{\mathfrak{h}}\left(X_{\sigma}\right) \subset \mathfrak{h} \wedge \mathfrak{g}$, where $X_{\sigma}:=$ $R_{\sigma}\left(\pi\left(\sigma^{-1}\right)\right) \in \mathfrak{g} \wedge \mathfrak{g}$. 
In case the multiplicative Poisson structure $\pi$ on $G$ is given by an $r$ matrix $r \in \mathfrak{g} \wedge \mathfrak{g}$ (satisfying the modified Yang-Baxter equation), i.e.,

$$
\pi(g)=L_{g} r-R_{g} r
$$

we have

$$
X_{\sigma}=R_{\sigma}\left(\pi\left(\sigma^{-1}\right)\right)=R_{\sigma}\left(L_{\sigma^{-1}} r-R_{\sigma^{-1}} r\right)=\operatorname{Ad}_{\sigma^{-1}}(r)-r .
$$

A closed subgroup $H$ being coisotropic with respect to $\pi$ is equivalent to

$$
\operatorname{ad}_{\mathfrak{h}}(r) \subset \mathfrak{h} \wedge \mathfrak{g}
$$

since

$$
\begin{aligned}
\pi(g h)-R_{h}(\pi(g)) & =L_{g h} r-R_{g h} r-R_{h}\left(L_{g} r-R_{g} r\right) \\
& =L_{g h} r-R_{g h} r-L_{g} R_{h} r+R_{h} R_{g} r=L_{g h} r-L_{g} R_{h} r \\
& =L_{g h}\left(r-L_{h^{-1}} R_{h} r\right)=L_{g h}\left(\mathrm{id}-\operatorname{Ad}_{h^{-1}}\right)(r)
\end{aligned}
$$

and $L_{g h}\left(\mathrm{id}-\operatorname{Ad}_{h^{-1}}\right)(r) \in L_{g h}(\mathfrak{h} \wedge \mathfrak{g})$ for all $(g, h) \in G \times H$ if and only if

$$
\left(\mathrm{id}-\operatorname{Ad}_{h^{-1}}\right)(r) \in \mathfrak{h} \wedge \mathfrak{g}
$$

for all $h \in H$.

Corollary 2. Given a Poisson-Lie group $(G, \pi)$ with $\pi$ defined by an $r$ matrix $r \in \mathfrak{g} \wedge \mathfrak{g}$, a $\pi$-coisotropic closed subgroup $H$ of $G$ is coisotropic with respect to $\pi_{\sigma}$ for $\sigma \in G$, if and only if $\operatorname{ad}_{\mathfrak{h}}\left(\operatorname{Ad}_{\sigma^{-1}}(r)\right) \subset \mathfrak{h} \wedge \mathfrak{g}$.

\section{Non-standard Poisson $\mathbb{C} P^{n}$.}

In this section, we construct via affine Poisson structures on $S U(n)$ some $S U(n)$-covariant Poisson structures on $\mathbb{C} P^{n-1}$ that represent the classical counterpart of the nonstandard quantum projective spaces $\mathbb{C} P_{q, c}^{n}[\mathrm{DiNo}]$.

Recall that the standard Poisson $S U(n)$ is defined (up to a constant multiple) by the Poisson 2-tensor $\pi(u)=\pi^{(n)}(u):=L_{u} r-R_{u} r$ determined by the $r$-matrix

$$
r:=\sum_{1 \leq i<j \leq n} X_{i j}^{+} \wedge X_{i j}^{-}
$$

where $X_{i j}^{+}=e_{i j}-e_{j i}, X_{i j}^{-}=i\left(e_{i j}+e_{j i}\right)$, and $e_{i j}$ are the matrix units. 
It is well known that $S U(n-1) \cong\{1\} \oplus S U(n-1)($ or $S U(n-1) \oplus\{1\})$ and

$$
U(n-1) \cong\left\{\operatorname{det}(u)^{-1} \oplus u: u \in U(n-1)\right\}
$$

are Poisson-Lie subgroups of $S U(n)$ and hence induce the 'standard' $S U(n)$ covariant Poisson structures $\rho=\rho^{(n)}$ (also called Bruhat Poisson structure [LuWe1]) and $\tau=\tau^{(n-1)}$ on the sphere

$$
\mathbb{S}^{2 n-1} \cong S U(n) /[\{1\} \oplus S U(n-1)]
$$

and the complex projective space

$$
\mathbb{C} P^{n-1} \cong S U(n) / U(n-1)
$$

respectively.

Theorem 3. The closed subgroup $U(n-1)$ of $S U(n)$ is coisotropic with respect to the (left) $S U(n)$-covariant affine Poisson structure $\pi_{\sigma_{c}}$ on $S U(n)$ defined by

$$
\sigma_{c}:=\left(\sqrt{c} e_{11}+\sqrt{1-c} e_{n 1}-\sqrt{1-c} e_{1 n}+\sqrt{c} e_{n n}\right)+\sum_{i=2}^{n-1} e_{i i} \in S U(n)
$$

with $c \in[0,1]$. Hence $\pi_{\sigma_{c}}$ induces a (left) $S U(n)$-covariant Poisson structure $\tau_{c}$ on $\mathbb{C} P^{n-1} \cong S U(n) / U(n-1)$.

Proof. We set $\sigma=\sigma_{c}$ for simplicity. It is easy to see that if the Poisson structure $\tau_{c}$ induced by $\pi_{\sigma_{c}}$ on $\mathbb{C} P^{n-1} \cong S U(n) / U(n-1)$ is well defined, then it is automatically (left) $S U(n)$-covariant since $\pi_{\sigma_{c}}$ is. Now since $U(n-1)$ is a Poisson-Lie subgroup and hence coisotropic with respect to $\pi$, we have

$$
\operatorname{ad}_{\mathfrak{u}(n-1)}(r) \subset \mathfrak{u}(n-1) \wedge \mathfrak{s u}(n),
$$

and hence only need to show that

$$
\operatorname{ad}_{\mathfrak{u}(n-1)}\left(\operatorname{Ad}_{\sigma^{-1}}(r)\right) \subset \mathfrak{u}(n-1) \wedge \mathfrak{s u}(n) .
$$

From

$$
\begin{cases}\operatorname{Ad}_{\sigma^{-1}}\left(X_{i j}^{+}\right)=X_{i j}^{+}, & \text {if } 1<i<j<n \\ \operatorname{Ad}_{\sigma^{-1}}\left(X_{1 j}^{+}\right)=\sqrt{c} X_{1 j}^{+}+\sqrt{1-c} X_{j n}^{+}, & \text {if } 1<j<n \\ \operatorname{Ad}_{\sigma^{-1}}\left(X_{i n}^{+}\right)=-\sqrt{1-c} X_{1 i}^{+}+\sqrt{c} X_{i n}^{+}, & \text {if } 1<i<n \\ \operatorname{Ad}_{\sigma^{-1}}\left(X_{1 n}^{+}\right)=X_{1 n}^{+}, & \end{cases}
$$


and

$$
\left\{\begin{array}{rlrl}
\operatorname{Ad}_{\sigma^{-1}}\left(X_{i j}^{-}\right)=X_{i j}^{-}, & & \text {if } 1<i<j<n \\
\operatorname{Ad}_{\sigma^{-1}}\left(X_{1 j}^{-}\right)=\sqrt{c} X_{1 j}^{-}-\sqrt{1-c} X_{j n}^{-}, & & \text {if } 1<j<n \\
\operatorname{Ad}_{\sigma^{-1}}\left(X_{i n}^{-}\right)=\sqrt{1-c} X_{1 i}^{-}+\sqrt{c} X_{i n}^{-}, & & \text {if } 1<i<n \\
\operatorname{Ad}_{\sigma^{-1}}\left(X_{1 n}^{-}\right)= & (2 c-1) X_{1 n}^{-} & & \\
& +2 i \sqrt{c(1-c)}\left(e_{11}-e_{n n}\right) . &
\end{array}\right.
$$

we get

$$
\begin{aligned}
& \operatorname{Ad}_{\sigma^{-1}}(r) \\
& =\sum_{1 \leq i<j \leq n} \operatorname{Ad}_{\sigma^{-1}}\left(X_{i j}^{+}\right) \wedge \operatorname{Ad}_{\sigma^{-1}}\left(X_{i j}^{-}\right) \\
& =\sum_{1<i<j<n} X_{i j}^{+} \wedge X_{i j}^{-}+X_{1 n}^{+} \wedge\left[(2 c-1) X_{1 n}^{-}+2 \sqrt{c(1-c)} i\left(e_{11}-e_{n n}\right)\right] \\
& \quad+\sum_{1<i<n}\left(\sqrt{c} X_{1 i}^{+}+\sqrt{1-c} X_{i n}^{+}\right) \wedge\left(\sqrt{c} X_{1 i}^{-}-\sqrt{1-c} X_{i n}^{-}\right) \\
& +\sum_{1<i<n}\left(-\sqrt{1-c} X_{1 i}^{+}+\sqrt{c} X_{i n}^{+}\right) \wedge\left(\sqrt{1-c} X_{1 i}^{-}+\sqrt{c} X_{i n}^{-}\right) \\
& =2(1-c) \sum_{1<i<j<n} X_{i j}^{+} \wedge X_{i j}^{-}+2 \sqrt{c(1-c)} X_{1 n}^{+} \wedge i\left(e_{11}-e_{n n}\right) \\
& \quad+(2 c-1) r+2 \sqrt{c(1-c)} \sum_{1<i<n}\left(X_{i n}^{+} \wedge X_{1 i}^{-}-X_{1 i}^{+} \wedge X_{i n}^{-}\right)
\end{aligned}
$$

which is in $(2 c-1) r+(\mathfrak{u}(n-1) \wedge \mathfrak{s u}(n))$, since

$$
X_{i j}^{+}, X_{i j}^{-}, X_{i n}^{+}, X_{i n}^{-}, i\left(e_{11}-e_{n n}\right) \in \mathfrak{u}(n-1)
$$

for all $1<i<j<n$. So we get

$$
\begin{aligned}
& \operatorname{ad}_{\mathfrak{u}(n-1)}\left(\operatorname{Ad}_{\sigma^{-1}}(r)\right) \\
& \quad \subset(2 c-1) \operatorname{ad}_{\mathfrak{u}(n-1)}(r)+\operatorname{ad}_{\mathfrak{u}(n-1)}(\mathfrak{u}(n-1) \wedge \mathfrak{s u}(n)) \\
& \quad \subset \mathfrak{u}(n-1) \wedge \mathfrak{s u}(n)
\end{aligned}
$$

because $\operatorname{ad}_{\mathfrak{u}(n-1)}(r) \subset \mathfrak{u}(n-1) \wedge \mathfrak{s u}(n)$ and

$$
\operatorname{ad}_{\mathfrak{u}(n-1)}(\mathfrak{u}(n-1) \wedge \mathfrak{s u}(n)) \subset \mathfrak{u}(n-1) \wedge \mathfrak{s u}(n) .
$$


The Poisson manifold $\left(\mathbb{C} P^{n-1}, \tau_{c}\right)$ with $c \in(0,1)$ is referred to as a nonstandard Poisson $\mathbb{C} P^{n-1}$. Note that $\tau_{1}=\tau^{(n-1)}$ the standard Poisson structure on $\mathbb{C} P^{n-1}$ since $\sigma_{1}=1 \in S U(n)$ and hence $\pi_{\sigma_{1}}=\pi$. On the other hand, $R_{\sigma_{0}}$ simply swaps the first column with the $n$-th column and hence $\tau_{0}$ is the standard Poisson structure on $\mathbb{C} P^{n-1} \cong$ $S U(n) /\left\{u \oplus \operatorname{det}(u)^{-1}: u \in U(n-1)\right\}$ induced by $\pi$.

We remark that the above nonstandard Poisson structures on $\mathbb{C} P^{n-1}$ form only a part of the one-parameter family of all $S U(n)$-covariant Poisson structures on $\mathbb{C} P^{n-1}$ that can be classified using the results of [KhRaRu] and [Kos2] as follows. (We thank the referee for providing this argument.) Indeed it is easy to see, as in [LuWe2], that the difference of two covariant Poisson 2-tensors is an invariant alternating 2-tensor. Applying the known fact that the $p$-th de Rham cohomology of the Grassmannian $G_{n}^{k}$ of $k$-dimensional subspaces in $\mathbb{C}^{n}$ is isomorphic to the space of $S U(n)$-invariant $p$-forms on $G_{n}^{k}$ [Kos2] to the case of $k=1$ and $p=2$, we see that the known de Rham cohomology group $H_{D R}^{2}\left(\mathbb{C} P^{n-1}\right) \cong \mathbb{R}$ (for $\left.n \geq 2\right)$ is isomorphic to the space of all $S U(n)$-invariant 2 -forms on $\mathbb{C} P^{n-1}$. So every $S U(n)$ invariant 2-form, or equivalently, every $S U(n)$-invariant 2-tensor (by duality via the standard $U(1)$-invariant Euclidean structure of $\mathbb{C}^{n}$ ) on $\mathbb{C} P^{n-1}$ is a scalar multiple of the $S U(n)$-invariant Fubini-Study symplectic form on the compact Kähler manifold $\mathbb{C} P^{n-1}$, or equivalently, the corresponding nondegenerate $S U(n)$-invariant 2-tensor $\tilde{\tau}$ on $\mathbb{C} P^{n-1}$. (In section 4, we give an elementary direct proof of this fact.) So every $S U(n)$-covariant Poisson 2 -tensor on $\mathbb{C} P^{n-1}$ belongs to the family $\tau_{1}+\mathbb{R} \tilde{\tau}$. On the other hand, it is easy to see that the sum of an $S U(n)$-covariant 2-tensor and an $S U(n)$ invariant 2-tensor is $S U(n)$-covariant. Furthermore it is known [KhRaRu] that the Schouten-Nijenhuis bracket $\left[\left[\tilde{\tau}, \tau_{1}\right]\right]=0$ and hence every 2-tensor in $\tau_{1}+\mathbb{R} \tilde{\tau}$ is a Poisson 2-tensor. (In fact, the covariance of $\tau_{1}$ implies that the 3-tensor $\left[\left[\tilde{\tau}, \tau_{1}\right]\right]$ is $S U(n)$-invariant. However since $H_{D R}^{3}\left(\mathbb{C} P^{n-1}\right)=0$, there is no non-zero $S U(n)$-invariant 3-tensor on $\mathbb{C} P^{n-1}$.) Thus $\tau_{1}+\mathbb{R} \tilde{\tau}$ consists of all $S U(n)$-covariant Poisson 2-tensors on $\mathbb{C} P^{n-1}$.

In [Dr2], Drinfeld associated a Lagrangian subalgebra of the double Lie algebra $\mathfrak{d}=\mathfrak{g} \bowtie \mathfrak{g}^{*}$ with each $G$-covariant Poisson structure $\tau$ on a homogeneous space $M$ of $G$. More precisely,

$$
\left.\mathfrak{i}_{m}:=\left\{(x, \xi) \in \mathfrak{g} \times\left(\mathfrak{g}_{m}\right)^{\perp}: x+\mathfrak{g}_{m}=\xi\right\lrcorner \tau(m)\right\}
$$

[Dr2, ELu] is the Lagrangian subalgebra associated with $(M, \tau)$ at a point $m \in M$, where $\mathfrak{g}_{m} \subset \mathfrak{g}$ is the Lie algebra of the stabilizer subgroup of $G$ at $m$, the coset space $\mathfrak{g} / \mathfrak{g}_{m}$ is identified with the tangent space $T_{m} M$ via 
the differential of the action $g \in G \mapsto g m \in M$ at the neutral element $e$, and $\left(\mathfrak{g}_{m}\right)^{\perp} \subset \mathfrak{g}^{*}$ consisting of linear functionals of $\mathfrak{g}$ that annihilate $\mathfrak{g}_{m}$ is identified with the cotangent space $T_{m}^{*} M$. Since the correspondence $m \mapsto \mathfrak{i}_{m}$ is $G$-equivariant [Dr2], $\mathfrak{i}_{m_{0}}$ for any specific $m_{0} \in M$ determines the other $\mathfrak{i}_{m}$. Interesting results have been obtained recently on this topic. For example, Karolinsky classified all Lagrangian subalgebras of the double Lie algebra $\mathfrak{s l}(n, \mathbb{C})=\mathfrak{s u}(n) \bowtie \mathfrak{s u}(n)^{*}[\mathrm{Ka}]$, and Evens and Lu studied the geometry of the variety of all Lagrangian subalgebras of $\mathfrak{s l}(n, \mathbb{C})[\mathrm{ELu}$. In the following, we identify explicitly the Lagrangian subalgebra associated with the $S U(n)$ covariant nonstandard Poisson $\left(\mathbb{C} P^{n-1}, \tau_{c}\right)$ at the point $[(1,0, . ., 0)]$, and we denote by $\left\{X_{+}^{i j}, X_{-}^{i j}\right\}_{1 \leq i<j \leq n} \cup\left\{E^{k}\right\}_{k=2}^{n} \subset \mathfrak{s u}(n)^{*}$ the basis dual to the basis $\left\{X_{i j}^{+}, X_{i j}^{-}\right\}_{1 \leq i<j \leq n} \cup\left\{i\left(e_{11}-e_{k k}\right)\right\}_{k=2}^{n} \subset \mathfrak{s u}(n)$.

Proposition 4. The Lagrangian subalgebra $\mathfrak{i}_{[(1,0, . ., 0)]}$ associated with the $S U(n)$-covariant nonstandard Poisson homogeneous space $\left(\mathbb{C} P^{n-1}, \tau_{c}\right)$ at the point $[(1,0, . ., 0)] \in \mathbb{C} P^{n-1}$ is the linear span of $\mathfrak{u}(n-1) \times\{0\}$ and the vectors $\left( \pm 2(c-1) X_{1 j}^{\mp}, X_{ \pm}^{1 j}\right)$ with $1<j \leq n$ in $\mathfrak{s u}(n) \bowtie \mathfrak{s u}(n)^{*}$.

Proof. The stabilizer subgroup of $G=S U(n)$ at $m=[(1,0, \ldots, 0)] \in \mathbb{C} P^{n-1}$ is $U(n-1)$, and the differential $\eta$ at $e \in S U(n)$ of the action map $g \in S U(n) \mapsto g[(1,0, \ldots, 0)] \in \mathbb{C} P^{n-1}$ sends $e$ to $[(1,0, \ldots, 0)]$ and identifies $\mathfrak{s u}(n) / \mathfrak{u}(n-1)$ with $T_{[(1,0, \ldots, 0)]} \mathbb{C} P^{n-1}$. Since $\tau_{c}([(1,0, \ldots, 0)])$ is the projection of $\pi_{\sigma_{c}}(e)$ under $\eta$, the Lagrangian subalgebra $\mathfrak{i}_{[(1,0, \ldots, 0)]}$ associated with $\left(\mathbb{C} P^{n-1}, \tau_{c}\right)$ at the point $[(1,0, \ldots, 0)]$ equals

$$
\begin{aligned}
& \left.\left\{(x, \xi) \in \mathfrak{s u}(n) \times \mathfrak{u}(n-1)^{\perp}: x \equiv \xi\right\lrcorner \pi_{\sigma_{c}}(e)(\bmod \mathfrak{u}(n-1))\right\} \\
& \left.=\left\{(\xi\lrcorner \pi_{\sigma_{c}}(e), \xi\right): \xi \in \mathfrak{u}(n-1)^{\perp}\right\}+\mathfrak{u}(n-1) \times\{0\}
\end{aligned}
$$

which is the linear span of $\mathfrak{u}(n-1) \times\{0\}$ and the vectors $\left.\left(X_{ \pm}^{1 j}\right\lrcorner \pi_{\sigma_{c}}(e), X_{ \pm}^{1 j}\right)$ with $1<j \leq n$ in $\mathfrak{s u}(n) \bowtie \mathfrak{s u}(n)^{*}$ because $\left\{X_{+}^{1 j}, X_{-}^{1 j}\right\}_{j=2}^{n}$ is a basis of $\mathfrak{u}(n-1)^{\perp}$. From the proof of the above theorem, 
we have

$$
\begin{aligned}
\pi_{\sigma_{c}}(e)= & R_{\alpha_{c}}\left(\pi\left(\sigma_{c}^{-1}\right)\right)=\operatorname{Ad}_{\sigma_{c}^{-1}}(r)-r \\
= & 2(1-c) \sum_{1<i<j<n} X_{i j}^{+} \wedge X_{i j}^{-}+2 \sqrt{c(1-c)} X_{1 n}^{+} \wedge i\left(e_{11}-e_{n n}\right) \\
& +2(c-1) r+2 \sqrt{c(1-c)} \sum_{1<i<n}\left(X_{i n}^{+} \wedge X_{1 i}^{-}-X_{1 i}^{+} \wedge X_{i n}^{-}\right) \\
= & 2(c-1)\left(\sum_{1<j \leq n} X_{1 j}^{+} \wedge X_{1 j}^{-}+\sum_{1<i<n} X_{i n}^{+} \wedge X_{i n}^{-}\right) \\
& +2 \sqrt{c(1-c)} X_{1 n}^{+} \wedge i\left(e_{11}-e_{n n}\right) \\
& +2 \sqrt{c(1-c)} \sum_{1<i<n}\left(X_{i n}^{+} \wedge X_{1 i}^{-}-X_{1 i}^{+} \wedge X_{i n}^{-}\right)
\end{aligned}
$$

and hence

$$
\left.X_{ \pm}^{1 j}\right\lrcorner \pi_{\sigma_{c}}(e) \equiv \pm 2(c-1) X_{1 j}^{\mp}(\bmod \mathfrak{u}(n-1))
$$

Thus the Lagrangian subalgebra $\mathfrak{i}_{[(1,0, . ., 0)]}$ is the linear span of $\mathfrak{u}(n-1) \times\{0\}$ and the vectors $\left( \pm 2(c-1) X_{1 j}^{\mp}, X_{ \pm}^{1 j}\right)$ with $1<j \leq n$ in $\mathfrak{s u}(n) \bowtie \mathfrak{s u}(n)^{*}$.

\section{Standard Poisson sphere in $\mathbb{C} P^{n}$.}

In this section, we show that the nonstandard Poisson $\left(\mathbb{C} P^{n-1}, \tau_{c}\right)$ contains a copy of the standard Poisson $\mathbb{S}^{2 n-3}$ generalizing the result of [LuWe2] for $n=1$.

We first remark that $X_{i j}^{+}, X_{i j}^{-}, X_{i n}^{+}, X_{i n}^{-} \in \mathfrak{s u}(n-1)$ but $i\left(e_{11}-e_{n n}\right) \notin$ $\mathfrak{s u}(n-1)$, so $\operatorname{ad}_{\mathfrak{s u}(n-1)}\left(\operatorname{Ad}_{\sigma^{-1}}(r)\right) \nsubseteq \mathfrak{s u}(n-1) \wedge \mathfrak{s u}(n)$ and hence $\pi_{\sigma_{c}}$ does not induce a Poisson structure on $\mathbb{S}^{2 n-1} \cong S U(n) / S U(n-1)$. On the other hand, as a generalization of Lu and Weinstein's result on covariant Poisson spheres $\mathbb{S}^{2}=\mathbb{C} P^{1}[\mathrm{LuWe}]$, we can show that $\left(\mathbb{C} P^{n-1}, \tau_{c}\right)$ contains a copy of the standard Poisson sphere $\left(\mathbb{S}^{2 n-3}, \rho^{(n-1)}\right)$. Here it is understood that $\rho^{(1)}=0$ on $\mathbb{S}^{1}$ by definition.

Theorem 5. The standard Poisson sphere $\left(\mathbb{S}^{2 n-3}, \rho^{(n-1)}\right)$ is embedded in $\left(\mathbb{C} P^{n-1}, \tau_{c}\right)$ for $c \in(0,1)$ and $n \geq 2$. 
Proof. Note that the quotient map $\phi: S U(n) \rightarrow \mathbb{C} P^{n-1}$ can be viewed as the composition of the quotient map

$$
\phi_{1}: u \in S U(n) \mapsto u_{1} \in \mathbb{S}^{2 n-1} \cong S U(n) / S U(n-1)
$$

and the quotient map

$$
\phi_{2}: v \in \mathbb{S}^{2 n-1} \mapsto[v] \in \mathbb{C} P^{n-1} \cong \mathbb{S}^{2 n-1} / \mathbb{T},
$$

where the circle group $\mathbb{T}$ acts diagonally on $\mathbb{S}^{2 n-1} \subset \mathbb{C}^{n}$ and

$$
u_{1}:=\left(u_{11}, u_{21}, \ldots, u_{n 1}\right) \in \mathbb{S}^{2 n-1} \subset \mathbb{C}^{n}
$$

is the first column of $u \in S U(n)$. It is well known that $\phi_{2}$ is a diffeomorphism from the submanifold

$$
S_{+}:=\left\{v \in \mathbb{S}^{2 n-1}: v_{1}>0\right\} \subset \mathbb{S}^{2 n-1}
$$

onto its image $\phi_{2}\left(S_{+}\right) \subset \mathbb{C} P^{n-1}$, and

$$
\phi_{3}: v \in S_{c} \mapsto \phi_{3}(v):=\frac{1}{\sqrt{1-c}}\left(v_{2}, \ldots, v_{n}\right) \in \mathbb{S}^{2 n-3}
$$

is a diffeomorphism identifying

$$
S_{c}:=\left\{v \in \mathbb{S}^{2 n-1}: v_{1}=\sqrt{c}\right\} \subset S_{+}
$$

with $\mathbb{S}^{2 n-3}$. We denote by $\psi: u \in S U(n) \mapsto u_{n} \in \mathbb{S}^{2 n-1}$ the projection to the last column. Functions similar to $\phi_{1}, \phi_{2}$, and $\psi$, for other dimensions than $n$, will be denoted by the same symbols for the simplicity of notation. First we assume that $n>2$. For each $v \in S_{c}$, we can find some $u^{\prime} \in$ $S U(n)$ with the first column $u_{1}^{\prime}=(1,0, \ldots, 0)$ and the last column $u_{n}^{\prime}=$ $\sqrt{1-c}^{-1}\left(0, v_{2}, \ldots, v_{n}\right)$. Note that the first row of $u^{\prime}$ has to be $(1,0, \ldots, 0)$, and hence

$$
u^{\prime}=1 \oplus u^{\prime \prime} \in\{1\} \oplus S U(n-1)
$$

for some $u^{\prime \prime} \in S U(n-1)$ with

$$
\left(u^{\prime \prime}\right)_{n-1}=\sqrt{1-c}^{-1}\left(v_{2}, \ldots, v_{n}\right)=\phi_{3}(v) .
$$

Furthermore since $\{1\} \oplus S U(n-1)$ is a Poisson-Lie subgroup of $S U(n)$,

$$
\pi\left(u^{\prime}\right)=0 \oplus \pi^{(n-1)}\left(u^{\prime \prime}\right) \in\{0\} \oplus \wedge^{2} T_{u^{\prime \prime}} S U(n-1) \subset \wedge^{2} T_{u^{\prime}} S U(n)
$$


where $\pi^{(n-1)}$ is the standard multiplicative Poisson structure on $S U(n-1)$. Note that

$$
\rho^{(n-1)}\left(\phi_{3}(v)\right)=(D \psi)_{u^{\prime \prime}}\left(\pi^{(n-1)}\left(u^{\prime \prime}\right)\right)
$$

for the standard Poisson 2-tensor $\rho^{(n-1)}$ on $\mathbb{S}^{2 n-3}$. Here we take

$$
\mathbb{S}^{2 n-3}=S U(n-1) /[S U(n-2) \oplus\{1\}] .
$$

For

$$
u:=R_{\sigma_{c}}\left(u^{\prime}\right)=u^{\prime} \sigma_{c} \in S U(n)
$$

we have

$$
\phi_{1}(u)=u_{1}=\left(u^{\prime} \sigma_{c}\right)_{1}=v \in S_{c}
$$

and in $\wedge^{2} T_{v} S_{+}$,

$$
\begin{aligned}
\left(D \phi_{1}\right)_{u}\left(\pi_{\sigma_{c}}(u)\right) & =\left(D \phi_{1}\right)_{u}\left(R_{\sigma_{c}}\left(\pi\left(u^{\prime}\right)\right)\right)=\left(D \phi_{1}\right)_{u}\left(\pi\left(u^{\prime}\right) \sigma_{c}\right) \\
& =\sqrt{1-c}(D \psi)_{u^{\prime}}\left(\pi\left(u^{\prime}\right)\right) \in \wedge^{2} T_{v} S_{c} \subset \wedge^{2} T_{v} S_{+}
\end{aligned}
$$

because the first columns of the component matrices in the 2-tensor $\pi\left(u^{\prime}\right)=$ $0 \oplus \pi^{(n-1)}\left(u^{\prime \prime}\right)$ are all zero. Note that

$$
\begin{aligned}
\tau_{c}([v]) & =\tau_{c}\left(\phi_{2}(v)\right)=\tau_{c}(\phi(u)) \\
& =(D \phi)_{u}\left(\pi_{\sigma_{c}}(u)\right)=\left(D \phi_{2}\right)_{\phi_{1}(u)}\left(\left(D \phi_{1}\right)_{u}\left(\pi_{\sigma_{c}}(u)\right)\right)
\end{aligned}
$$

is a well-defined 2-tensor at $[v] \in \phi_{2}\left(S_{c}\right) \subset \mathbb{C} P^{n-1}$ and $\phi_{2}$ is a diffeomorphism on $S_{+}$. So

$$
\pi^{\prime}: v \in S_{c} \mapsto\left(D \phi_{1}\right)_{u}\left(\pi_{\sigma_{c}}(u)\right) \in \wedge^{2} T_{v} S_{c}
$$

is a well-defined Poisson 2-tensor on $S_{c}$ and $\phi_{2}\left(S_{c}\right)$ is a Poisson submanifold of $\left(\mathbb{C} P^{n-1}, \tau_{c}\right)$ that is Poisson isomorphic to $\left(S_{c}, \pi^{\prime}\right)$. Under the diffeomorphism $\phi_{3}: S_{c} \rightarrow \mathbb{S}^{2 n-3}$ identifying $v \in S_{c}$ with $\phi_{3}(v) \in \mathbb{S}^{2 n-3}$, the 2-tensor $\pi^{\prime}(v)$ is identified with

$$
\begin{aligned}
\left(D \phi_{3}\right)_{v}\left(\left(D \phi_{1}\right)_{u}\left(\pi_{\sigma_{c}}(u)\right)\right) & =\left(D \phi_{3}\right)_{v}\left(\sqrt{1-c}(D \psi)_{u^{\prime}}\left(\pi\left(u^{\prime}\right)\right)\right) \\
& =\left(D \phi_{3}\right)_{v}\left(\sqrt{1-c}\left(0 \oplus(D \psi)_{u^{\prime}}\left(\pi^{(n-1)}\left(u^{\prime \prime}\right)\right)\right)\right) \\
& =\left(D \phi_{3}\right)_{v}\left(\sqrt{1-c}\left(0 \oplus \rho^{(n-1)}\left(\phi_{3}(v)\right)\right)\right) \\
& =\rho^{(n-1)}\left(\phi_{3}(v)\right) \in \wedge^{2} T_{\phi_{3}(v)} \mathbb{S}^{2 n-3} .
\end{aligned}
$$

Thus $\left(S_{c}, \pi^{\prime}\right)$ or $\left(\phi_{2}\left(S_{c}\right), \tau_{c}\right)$ is Poisson isomorphic to the standard Poisson sphere $\left(\mathbb{S}^{2 n-3}, \rho^{(n-1)}\right)$. When $n=2$, for $v \in S_{c}$ with $v_{2} \neq \sqrt{1-c}$, we cannot 
find a $u^{\prime} \in S U(2)$ with the first column $u_{1}^{\prime}=(1,0)$ and the last column $u_{2}^{\prime}=\sqrt{1-c}^{-1}\left(0, v_{2}\right)$. But for $v_{0}=(\sqrt{c}, \sqrt{1-c})$, such a $u_{0}^{\prime}$ exists, namely, $u_{0}^{\prime}=I_{2}$ the $2 \times 2$ identity matrix, and the above argument essentially works. More precisely, it is well known that $\pi\left(u_{0}^{\prime}\right)=0$ since $u_{0}^{\prime} \in U(1) \subset S U(2)$, and hence for $u_{0}=u_{0}^{\prime} \sigma_{c}=\sigma_{c}$,

$$
\left(D \phi_{1}\right)_{u_{0}}\left(\pi_{\sigma_{c}}\left(u_{0}\right)\right)=\left(D \phi_{1}\right)_{u_{0}}\left(R_{\sigma_{c}}\left(\pi\left(u_{0}^{\prime}\right)\right)\right)=\left(D \phi_{1}\right)_{u_{0}}(0)=0 .
$$

So

$$
\tau_{c}\left(\left[v_{0}\right]\right)=\left(D \phi_{2}\right)_{v_{0}}\left(D \phi_{1}\right)_{u_{0}}\left(\pi_{\sigma_{c}}\left(u_{0}\right)\right)=0 .
$$

On the other hand, since $\tau_{c}$ on $\mathbb{C} P^{1} \approx \mathbb{S}^{2}$ is $S U(2)$-covariant and $U(1) \subset$ $S U(2)$ consists of 0-dimensional leaves, the action of any

$$
t=\left(\begin{array}{cc}
e^{i \theta} & 0 \\
0 & e^{-i \theta}
\end{array}\right) \in U(1)
$$

on $\mathbb{C} P^{1}$ preserves the Poisson structure $\tau_{c}$. In particular, $\tau_{c}\left(\left[t v_{0}\right]\right)=0$ for any $t \in U(1)$. Since any $v=\left(\sqrt{c}, \sqrt{1-c} e^{i \theta}\right) \in S_{c}$ is equivalent to a $t v_{0}$ with $t \in U(1)$ under the diagonal $\mathbb{T}$-action, namely,

$$
\begin{aligned}
{[v] } & =\left[\left(\begin{array}{c}
e^{-i \theta / 2} \sqrt{c} \\
e^{-i \theta / 2} \sqrt{1-c} e^{i \theta}
\end{array}\right)\right]=\left[\left(\begin{array}{c}
e^{-i \theta / 2} \sqrt{c} \\
e^{i \theta / 2} \sqrt{1-c}
\end{array}\right)\right] \\
& =\left[\left(\begin{array}{cc}
e^{-i \theta / 2} & 0 \\
0 & e^{i \theta / 2}
\end{array}\right)\left(\begin{array}{c}
\sqrt{c} \\
\sqrt{1-c}
\end{array}\right)\right]=\left[\left(\begin{array}{cc}
e^{-i \theta / 2} & 0 \\
0 & e^{i \theta / 2}
\end{array}\right) v_{0}\right]
\end{aligned}
$$

in $\mathbb{C} P^{1}$, we have $\tau_{c}([v])=0$ for all $v \in S_{c}$, i.e., $\tau_{c}=0$ on $\phi_{2}\left(S_{c}\right) \subset \mathbb{C} P^{1}$. Since $\phi_{2}\left(S_{c}\right)$ is diffeomorphic to $S_{c}$ and hence to $\mathbb{S}^{1}$, we get the standard (trivial) Poisson $\left(\mathbb{S}^{1}, \rho^{(1)}\right)$ embedded in $\left(\mathbb{C} P^{1}, \tau_{c}\right)$.

\section{Invariant 2-tensor on $\mathbb{S}^{2 n-1}$.}

In this section, we first classify the $S U(n)$-invariant (contravariant alternating) 2-tensor on $\mathbb{S}^{2 n-1}$, and then we conclude that the canonical $S U(n)$ invariant symplectic structure on $\mathbb{C} P^{n-1}$ gives the only, up to a constant factor, $S U(n)$-invariant (contravariant alternating) 2-tensor on $\mathbb{C} P^{n-1}$.

For each $p \in \mathbb{S}^{2 n-1}$, we have $i p \in T_{p} \mathbb{S}^{2 n-1}$, and the orthogonal complement $E_{p}:=\{p, i p\}^{\perp} \subset T_{p} \mathbb{S}^{2 n-1}$ is a complex subspace of $\mathbb{C}^{n}=T_{p} \mathbb{C}^{n}$ endowed with a canonical symplectic structure $\tilde{\Omega}_{p}$ determined by the complex hermitian structure on $\mathbb{C}^{n}$. Indeed $(d \omega)_{p}=\tilde{\Omega}_{p}$ on $E_{p}$ for the unique 1 -form $\omega$, the standard contact structure, on $\mathbb{S}^{2 n-1}$ such that $\omega_{p}(i p)=1$ and 
$\omega_{p}\left(E_{p}\right)=\{0\}$ at each $p \in \mathbb{S}^{2 n-1}$. The contact manifold $\left(\mathbb{S}^{2 n-1}, \omega\right)$ with the diagonal $\mathbb{T}$-action on $\mathbb{S}^{2 n-1}$ is the standard prequantization [Kos1, We1] of the canonical $S U(n)$-invariant symplectic structure on $\mathbb{C} P^{n-1} \cong \mathbb{S}^{2 n-1} / \mathbb{T}$.

Since the vector fields $p \mapsto p$ and $p \mapsto i p$ on $\mathbb{S}^{2 n-1}$ are invariant under the $U(n)$-action, so is the distribution $p \mapsto E_{p}$ of tangent subspaces. Furthermore, since the $U(n)$-action preserves the complex hermitian structure on $\mathbb{C}^{n}$ (and on $E_{p}$ ), the field $p \mapsto \tilde{\Omega}_{p}$ of symplectic forms on $\mathbb{S}^{2 n-1}$ is also invariant under the $U(n)$-action. Thus the contravariant 2-tensor $\tilde{\pi}$ on $\mathbb{S}^{2 n-1}$ uniquely determined by the form $\tilde{\Omega}$ on $E \subset T S U(n)$ is $U(n)$-invariant. Note that this 2-tensor $\tilde{\pi}$ on $\mathbb{S}^{2 n-1}$, invariant under the diagonal $\mathbb{T}$-action, induces the cnanonical symplectic structure on $\mathbb{C} P^{n-1} \cong \mathbb{S}^{2 n-1} / \mathbb{T}$ determined by its complex hermitian structure.

Given an $S U(n)$-invariant contravriant 2-tensors $\pi \neq 0$ on $\mathbb{S}^{2 n-1}$ with $n \geq 2$, we show that $\pi=\tilde{\pi}$ after a suitable normalization if $n \neq 3$ or if $\pi$ is $\bar{U}(n)$-invariant. Through the standard Euclidean structure on $\mathbb{C}^{n} \cong \mathbb{R}^{2 n}$, we identify the $S U(n)$-invariant contravriant 2-tensors $\pi \neq 0$ on $\mathbb{S}^{2 n-1}$ with $S U(n)$-invariant 2 -forms $\Omega \neq 0$ on $\mathbb{S}^{2 n-1}$.

First we show that the tangent vector

$$
e_{1}^{\prime}:=i e_{1} \in T_{e_{1}} \mathbb{S}^{2 n-1}=i \mathbb{R} \oplus \mathbb{C}^{n-1}
$$

at $e_{1} \in \mathbb{S}^{2 n-1}$ is in

$$
\operatorname{ker} \Omega_{p}:=\left\{v \in T_{p} \mathbb{S}^{2 n-1}: \Omega_{p}(v, \cdot)=0\right\} .
$$

If not, then we can find an orthonormal set $\left\{e_{i}^{\prime}\right\}_{i=2}^{n-1} \cup\left\{\eta_{i}^{\prime}\right\}_{i=1}^{n} \subset 0 \oplus \mathbb{C}^{n-1}$ such that $\Omega_{e_{1}}\left(e_{i}^{\prime}, \eta_{j}^{\prime}\right)=\delta_{i j} a_{i i}$ and $\Omega_{e_{1}}\left(e_{i}^{\prime}, e_{j}^{\prime}\right)=\Omega_{e_{1}}\left(\eta_{i}^{\prime}, \eta_{j}^{\prime}\right)=0$ with $a_{i i} \in \mathbb{R}$ and $a_{11} \neq 0$. Now since $\Omega$ is $S U(n)$-invariant, we have

$$
\Omega_{e_{1}}\left(e_{1}^{\prime}, u\left(\eta_{1}^{\prime}\right)\right)=\Omega_{u\left(e_{1}\right)}\left(u\left(e_{1}^{\prime}\right), u\left(\eta_{1}^{\prime}\right)\right)=\Omega_{e_{1}}\left(e_{1}^{\prime}, \eta_{1}^{\prime}\right)=a_{11}
$$

for any $u \in\{1\} \oplus S U(n-1) \subset S U(n)$. This cannot be true, since by a suitable choice of $u, u\left(\eta_{1}^{\prime}\right)$ can be any unit vector in $0 \oplus \mathbb{C}^{n-1}$, for example, $\eta_{n}^{\prime}$. Thus $e_{1}^{\prime}=i e_{1} \in \operatorname{ker} \Omega_{p}$.

Now with respect to the standard orthonormal $\mathbb{R}$-linear basis of

$$
i \mathbb{R} \oplus \mathbb{R}^{n-1} \oplus \mathbb{R}^{n-1} \cong i \mathbb{R} \oplus \mathbb{C}^{n-1}=T_{e_{1}} \mathbb{S}^{2 n-1}
$$

the 2-form $\Omega_{e_{1}}$ can be represented by a block diagonal matrix $0 \oplus B$ where $B \in M_{2(n-1)}(\mathbb{R})$ is a skew symmetric matrix. The $S U(n)$-invariance of $\Omega \neq 0$ implies that $\Omega_{e_{1}} \neq 0$ and

$$
u B u^{-1}=u B u^{t}=B \neq 0,
$$


or $u B=B u$, for any $u \in S U(n-1) \subset O_{2 n-2}(\mathbb{R})$ since $1 \oplus u \in S U(n)$ and $(1 \oplus u)\left(e_{1}\right)=e_{1}$. (If $\Omega$ is $U(n)$-invariant, then $u B=B u$ for any $u \in U(n-1)$ since $1 \oplus u \in U(n)$.)

We claim that $B$ must be conformal, i.e., $\|B(v)\|=\|B\|\|v\|$ for all $v \in \mathbb{R}^{2 n-2}$ where $\|B\|:=\sup _{\|v\|=1}\|B(v)\|>0$. Let $w$ be a unit vector with $\|B(w)\|=\|B\|$. Since $S U(n-1)$ acts on $\mathbb{S}^{2 n-3} \subset \mathbb{R}^{2 n-2}$ transitively, for any unit vector $v \in \mathbb{R}^{2 n-2}$, we can find $u \in S U(n-1)$ with $u^{-1}(v)=w$, and hence

$$
\|B(v)\|=\left\|u B u^{-1}(v)\right\|=\|u(B(w))\|=\|B(w)\|=\|B\| .
$$

Thus $B /\|B\|$ is a skew-symmetric isometry on $\mathbb{R}^{2 n-2}$ and so $B /\|B\| \epsilon$ $\mathrm{O}_{2 n-2}(\mathbb{R})$.

If $n=2$, then any skew symmetric $0 \neq B /\|B\| \in O_{2}(\mathbb{R})$ determines the same 2 -form $\Omega_{e_{1}}$ on $0 \oplus \mathbb{R}^{2}$ and hence on $i \mathbb{R} \oplus \mathbb{R}^{2}$, up to a constant multiple. So $\Omega=\tilde{\Omega}$ after normalized.

If $n \geq 4$, then the commutativity of $\mathbb{T}^{n-2} \subset S U(n-1)$ with $B$ implies that $B$ is complex linear on $\mathbb{R}^{2 n-2}=\mathbb{C}^{n-1}$ and so $B /\|B\| \in U(n-1)$. In fact, since for any $1 \leq j \neq k \leq n-1, t_{j k \theta} B=B t_{j k \theta}$ for all $\theta \in \mathbb{R}$ implies that $B_{j j}, B_{k k} \in \mathbb{C}$ and $B_{k l}=0$ for any $j \neq l \neq k$, where $B=\left(B_{j k}\right)_{1 \leq j, k \leq n-1}$ with $B_{j k} \in M_{2}(\mathbb{R})$, and

$$
t_{j k \theta}:=e^{i \theta} e_{j j}+e^{-i \theta} e_{k k}+\sum_{\substack{1 \leq l \leq n-1 \\ j \neq l \neq k}} e_{l l} \in \mathbb{T}^{n-2} \subset S U(n-1) .
$$

It is well known that only scalar matrices in $M_{n-1}(\mathbb{C})$ commute with $S U(n-1)$, so we get $B /\|B\| \in \mathbb{T}$ with $-B /\|B\|=(B /\|B\|)^{*}=$ $(B /\|B\|)^{-1}$, i.e., $(B /\|B\|)^{2}=-1$ or $B= \pm i\|B\|$. Thus

$$
\Omega_{e_{1}}= \pm\|B\| \tilde{\Omega}_{e_{1}}
$$

a (real) constant multiple of the standard symplectic form. Hence we get $\pi=\tilde{\pi}$ after a suitable normalization.

If $\Omega$ is $U(n)$-invariant, then the commutativity of $\mathbb{T}^{n-1} \subset U(n-1)$ with $B$ implies that $B$ is complex linear and hence $B /\|B\| \in U(n-1)$ and as above, $\Omega= \pm\|B\| \tilde{\Omega}$. In fact, $t_{k \theta}^{\prime} B=B t_{k \theta}^{\prime}$ for all $\theta \in \mathbb{R}$ implies that $B_{k k} \in \mathbb{C}$ and $B_{k l}=0$ for any $l \neq k$, where

$$
t_{k \theta}^{\prime}:=e^{i \theta} e_{k k}+\sum_{\substack{1 \leq l \leq n-1 \\ l \neq k}} e_{l l} \in \mathbb{T}^{n-1} \subset U(n-1) .
$$


We observe that the quotient map $\phi: \mathbb{S}^{2 n-1} \rightarrow \mathbb{C} P^{n-1}$ and its differential $D \phi: T \mathbb{S}^{2 n-1} \rightarrow T \mathbb{C} P^{n-1}$ are $U(n)$-equivariant since the diagonal $\mathbb{T}$-action commutes with the $U(n)$-action. Furthermore, the restriction

$$
\left.(D \phi)\right|_{E}: E \rightarrow T \mathbb{C} P^{n-1}
$$

of $D \phi$ to the $U(n)$-equivariant subbundle $E$ defined above is a bundle isomorphism. So any $S U(n)$-invariant (and hence $U(n)$-invariant) 2-tensor $\tau \in \Gamma\left(\wedge^{2} T \mathbb{C} P^{n-1}\right)$ on $\mathbb{C} P^{n-1}$ can be 'pulled back' to an $U(n)$-invariant 2 -tensor

$$
\pi=\left.(D \phi)\right|_{E} ^{-1}(\tau) \in \Gamma\left(\wedge^{2} E\right) \subset \Gamma\left(\wedge^{2} T \mathbb{S}^{2 n-1}\right)
$$

on $\mathbb{S}^{2 n-1}$ which must be, up to a constant factor, equal to $\tilde{\pi}$. Thus $\tau=\tilde{\tau}:=$ $(D \phi)(\tilde{\pi})$ which is the standard symplectic 2 -tensor on $\mathbb{C} P^{n-1}$.

\section{References.}

[DaSo] P. Dazord and D. Sondaz, Groupes de Poisson affines, in 'Symplectic Geometry, Groupoids, and Integrable Systems', P. Dazord and A. Weinstein (Eds.), Springer-Verlag, 1991.

[DiNo] M.S. Dijkhuizen and M. Noumi, A family of quantum projective spaces and related $q$-hypergeometric orthogonal polynomials, preprint.

[Dr1] V.G. Drinfeld, Quantum groups, Proc. I.C.M. Berkeley 1986, 1, 789-820, Amer. Math. Soc., Providence, 1987.

[Dr2] V.G. Drinfeld, On Poisson homogeneous spaces of Poisson Lie groups, Theo. Math. Phys., 95 (1993), 226-227.

[ELu] S. Evens and J.H. Lu, On the variety of Lagrangian subalgebras, preprint, math.DG/9909005.

[Ka] E. Karolinsky, The classification of Poisson homogeneous spaces of compact Poisson Lie groups, Mathematical Physics, Analysis, and Geometry, 3 (1996), 272-289.

[KhRaRu] S. Khoroshkin, A. Radul and V. Rubtsov, A family of Poisson structures on hermitian symmetric spaces, Comm. Math. Phys., 152 (1993), 299-315. 
[KoVa] L.I. Korogodsky and L.L. Vaksman, Quantum G-spaces and Heisenberg algebra, in 'Quantum Groups', P.P. Kulish (Ed.), Lecture Notes in Mathematics, 1510, Springer-Verlag, Berlin, (1992), 56-66.

[Kos1] B. Kostant, Quantization and unitary representations. I: Prequantization, in 'Lectures in Modern Analysis and Applications, III', E.T. Taam (Ed.), Lecture Notes in Mathematics, 170, Springer-Verlag, Berlin and New York, (1970), 87-208.

[Kos2] B. Kostant, Lie algebra cohomology and generalized Schubert cells, Ann. of Math., 77 (1963), 72-144.

[Lu] J.H. Lu, Multiplicative and affine Poisson structures on Lie groups, $\mathrm{Ph}$. D. thesis, Univ. of California, Berkeley, 1990.

[LuWe1] J.H. Lu and A. Weinstein, Poisson Lie groups, dressing transformations and Bruhat decompositions, J. Diff. Geom., 31 (1990), $501-526$.

[LuWe2] J.H. Lu and A. Weinstein, Classification of SU(2)-covariant Poisson structures on $\mathbb{S}^{2}$, Comm. Math. Phys., 135 (1991), 229231.

[Po] P. Podles, Quantum spheres, Letters Math. Phys., 14 (1987), 193-202.

[Re] J. Renault, A Groupoid Approach to $C^{*}$-algebras, Lecture Notes in Mathematics, 793, Springer-Verlag, New York, 1980.

[RTF] N.Yu. Reshetikhin, L.A. Takhtadzhyan and L.D. Faddeev, Quantization of Lie groups and Lie algebras, Leningrad Math. J., 1 (1990), 193-225.

[Ri] M.A. Rieffel, Non-compact quantum groups associated with abelian subgroups, Comm. Math. Phys., 171 (1995), 181-201.

[Sh1] A.J.L. Sheu, Quantization of the Poisson SU(2) and its Poisson homogeneous space - the 2-sphere, Comm. Math. Phys., 135 (1991), 217-232.

[Sh2] A.J.L. Sheu, Compact quantum groups and groupoid $C^{*}$-algebras, J. Func. Anal., 144 (1997), 371-393. 
[Sh3] A.J.L. Sheu, Groupoid approach to quantum projective spaces, preprint.

[So] Ya.S. Soibelman, The algebra of functions on a compact quantum group, and its representations, Algebra Analiz., 2 (1990), 190221 (Leningrad Math. J., 2 (1991), 161-178).

[VaSo] L.L. Vaksman and Ya.S. Soibelman, The algebra of functions on the quantum group $S U(n+1)$, and odd-dimensional quantum spheres, Leningrad Math. J., 2 (1991), 1023-1042.

[We1] A. Weinstein, Lectures on Symplectic Manifolds, CBMS Regional Conference Series in Mathematics, 29, Amer. Math. Soc., Providence, 1977.

[We2] A. Weinstein, The local structure of Poisson manifolds, J. Diff. Geom., 18 (1983), 523-557.

[We3] A. Weinstein, Affine Poisson structures, International J. Math., 1 (1990), 343-360.

[Wo1] S.L. Woronowicz, Twisted SU(2) group: an example of a noncommutative differential calculus, Publ. RIMS., 23 (1987), 117181.

[Wo2] S.L. Woronowicz, Compact matrix pseudogroups, Comm. Math. Phys., 111 (1987), 613-665.

Department of Mathematics

UNIVERSITY OF KANSAS

LAWRENCE, KS 66045

E-mail address: sheu@falcon.cc.ukans.edu

Received OCtober 11, 1999 and Revised February 24, 2000. 